\title{
R-Matrix Arising from Affine Hecke Algebras and its Application to Macdonald's Difference Operators
}

\author{
Shin-ichi Kato \\ Division of Mathematics, Department of Fundamental Sciences, Faculty of Integrated Human Stud- \\ ies, Kyoto University, Kyoto, 606-01, Japan. e-mail address: kato@platon.kula.kyoto-u.ac.jp
}

Received: 25 May 1993 / in revised form: 7 January 1994

\begin{abstract}
We shall give a certain trigonometric R-matrix associated with each root system by using affine Hecke algebras. From this R-matrix, we derive a quantum Knizhnik-Zamolodchikov equation after Cherednik, and show that the solutions of this $\mathrm{KZ}$ equation yield eigenfunctions of Macdonald's difference operators.
\end{abstract}

In this paper, we shall construct the trigonometric R-matrix from affine Hecke algebras. Our R-matrix, which is obtained for each finite or affine root system, satisfies the Yang-Baxter equations in a generalized sense [C2] (see Theorem 2.4 and Proposition 3.6 below). As an application, we define a kind of quantum KnizhnikZamolodchikov equations (QKZ) following [C4]. Moreover, by using these equations, we shall show that Macdonald's difference operators (MDO) [Mac3] enter in the context of affine Hecke algebras through our R-matrix (Theorem 4.6). This result may be viewed as a certain $q$-analogue of Matsuo's correspondence [Mato] between "classical" Knizhnik-Zamolodchikov equations and zonal spherical systems (see also [C3]).

The contents of this paper is as follows. For a finite root system, we consider the affine Weyl group and its Hecke algebra simultaneously in $\operatorname{End}_{\mathbb{C}}(V)$ of some vector space $V$ and give the affine Weyl group actions in two ways on $V^{\sim}$, a certain extension of $V$, in Sect. 1. Then we define our R-matrix for the root system as a "difference" of these two actions (see 2.1), and show that they satisfy the Yang-Baxter equations (Theorem 2.4). Moreover, after the idea of Cherednik [C4], we extend our R-matrix to the affine root system by using $q$-shift operators in Sect. 3. As a result, we get the Heisenberg-Weyl group (an extension of the affine Weyl group) action on $V^{\sim}$ in two ways (see 3.10), and obtain QKZ. In Sect. 4, we review the definition of MDO, and show how the solutions to our QKZ give eigenfunctions of these MDO in Theorem 4.6. Section 5 is devoted to the proof of this theorem.

It should be noted that Cherednik announced similar results on QKZ and MDO [C5] which overlap with ours in Sects. 3-4 in part. There essentially the same QKZ is given, and more general eigenvalue problems than ours are discussed. Actually he gave an equivallence between $\mathrm{QKZ}$ and certain eigenvalue problems. Then he showed that Macdonald's original operators arise in his theory for the case of type 
$A$, but left open the other cases. ${ }^{1}$ Since we handle each MDO in an explicit way, our results on MDO (Theorem 4.6) may be viewed as an answer to this problem. It is also noted that the notion of admissible pairs of root systems in [Mac3] fits naturally in our formulation (see 4.3). Our way to derive QKZ is along Cherednik's method in [C4] using the R-matrix. But we hope the publication of this article will be a help to the understanding of this subject since we employ a somewhat different formulation from Cherednik's.

\section{Affine Weyl Groups and Affine Hecke Algebras}

In this section, we shall realize affine Weyl groups and their Hecke algebras in some endomorphism algebras. See [B, Matm, K and L] for basic references on root systems, affine Weyl groups and their Hecke algebras.

1.1. Let $\Delta$ be a reduced root system in a finite dimensional real Euclidean space $E$ with the inner product $\langle$,$\rangle . We choose and fix the set of positive roots \Delta^{+}$relative to some order and the set of simple roots $\Pi$ in $\Delta^{+}$.

Let $P$ be the weight lattice, $P=\left\{\lambda \in E \mid\left\langle\lambda, \alpha^{\vee}\right\rangle \in \mathbb{Z}(\forall \alpha \in \Delta)\right\}$ and $Q$ the root lattice of $\Delta$. Here $\alpha^{\vee}=2 \alpha /\langle\alpha, \alpha\rangle$, the coroot corresponding to $\alpha \in \Delta$. Let $W=W(\Delta)$ be the Weyl group of $\Delta$. The group $W$ acts on both $P$ and $Q$. We fix $L$ a sublattice of $P$ containing $Q$. Since the induced action of $W$ on $P / Q$ is trivial, $W$ also acts on $L$.

Set $A=\mathbb{C}[L]$, the group algebra of $L$ over $\mathbb{C}$. We denote by $e^{\lambda}$ the element of $A$ corresponding to $\lambda \in L$ as usual. The Weyl group $W$ acts on $A$ as $w\left(e^{\lambda}\right)=e^{w \lambda}(w \in$ $W, \lambda \in L)$. Let $V$ be the left $A$-module of rank $|W|$ with free basis $h_{w}(w \in W)$. Each element $F$ of $V$ can be written uniquely as $F=\sum_{w \in W} f_{w} h_{w}\left(f_{w} \in A\right)$.

Now we define several operators in $\operatorname{End}_{\mathbb{C}}(V)$ as follows.

1.2. The operator $f(f \in A)$ : Every element $f$ of $A$ acts on $V$ by left multiplication $F \rightarrow f F(F \in V)$.

We often regard $A$ as a subalgebra of $\operatorname{End}_{\mathbb{C}}(V)$ under this correspondence.

\subsection{The operator $r_{w}(w \in W)$ : The Weyl group $W$ acts on $V$ by}

$$
r_{w}\left(f h_{y}\right)=w(f) h_{w y}(f \in A, w, y \in W) .
$$

We note that the above $r_{w}(w \in W)$ and $A$ generate a subalgebra of $\operatorname{End}_{\mathbb{C}}(V)$, which is isomorphic to the group algebra $\mathbb{C}\left[W_{L}\right]$ of the extended affine Weyl group $W_{L}=W \ltimes L$ (the semidirect product of $L$ and $W$ ). We identify this subalgebra with $\mathbb{C}\left[W_{L}\right]$ and regard $V$ as a $\mathbb{C}\left[W_{L}\right]$-module. Note also that the action of $\mathbb{C}\left[W_{L}\right]$ on $V$ can be naturally identified with the left regular action of $\mathbb{C}\left[W_{L}\right]$.

1.4. We introduce a set of positive real parameters $t=\left\{t_{\alpha}, t_{\alpha}^{\prime}\right\}_{\alpha \in \Delta}$ satisfying

$$
t_{\alpha}=t_{\beta} \quad t_{\alpha}^{\prime}=t_{\beta}^{\prime} \quad \text { for } \quad \alpha, \beta \in \Delta \quad \text { with } \quad W \alpha=W \beta
$$

and

$$
t_{\alpha}=t_{\alpha}^{\prime} \quad \text { if } \quad\left\langle\alpha^{\vee}, L\right\rangle=\mathbb{Z}
$$

\footnotetext{
1 After we submitted this paper, we learned that Cherednik announced the solution of this remaining problem in his paper "The Macdonald constant term conjecture" (Internat. Math. Res. Notices No. 6, 165-177, 1993). See also his preprint "Induced representations of double affine Hecke algebras and applications."
} 
Let $s_{\alpha}$ be the reflection in $W$ corresponding to $\alpha \in \Delta$. Set $S=\left\{s_{\alpha}(\alpha \in \Pi)\right\}$, the set of simple reflections. We set $\alpha(s)=\alpha$ if $s=s_{\alpha}$ for $s \in S$ and $\alpha \in \Pi$.

1.5. The operator $T_{S}(s \in S)$ : The operator $T_{s}$ is defined by the following formulas:

$$
\begin{aligned}
T_{s}\left(h_{y}\right) & =h_{s y} & & \text { if } \quad y^{-1} \alpha>0 \\
& =\left(t_{\alpha}-1\right) h_{y}+t_{\alpha} h_{s y} & & \text { if } \quad y^{-1} \alpha<0,
\end{aligned}
$$

where $\alpha=\alpha(s)$ and

$$
\begin{aligned}
T_{s}\left(f h_{y}\right)-s(f) T_{s}\left(h_{y}\right)=\left(t_{\alpha}-1\right) \frac{f-s(f)}{1-e^{-\alpha}} h_{y} & \text { if }\left\langle\alpha^{\vee}, L\right\rangle=\mathbb{Z} ; \\
\quad=\left\{\left(t_{\alpha}-1\right)+e^{-\alpha} t_{\alpha}^{1 / 2}\left(t_{\alpha}^{\prime 1 / 2}-t_{\alpha}^{\prime-1 / 2}\right)\right\} \frac{f-s(f)}{1-e^{-2 \alpha}} h_{y} & \text { if }\left\langle\alpha^{\vee}, L\right\rangle=2 \mathbb{Z} ;
\end{aligned}
$$

for $y \in W$ and $f \in A$. (See [L]; cf. [Matm].) The formula (1.5b) in the case $\left\langle\alpha^{\vee}, L\right\rangle=2 \mathbb{Z}$ is also valid for $\alpha \in \Pi$ with $\left\langle\alpha^{\vee}, L\right\rangle=\mathbb{Z}$ by our assumption on $t_{\alpha}^{\prime}$ in 1.4. It is easily seen that the right-hand side of (1.5b) is actually in $V$.

Now we recall the definition of the affine Hecke algebra $H_{L}$. The affine Hecke algebra $H_{L}$ is the $\mathbb{C}$-algebra generated by elements $\mathscr{J}_{w}(w \in W)$ and $\theta_{\lambda}(\lambda \in L)$ with respect to the following relations:

$$
\begin{array}{cc}
\mathscr{J}_{s} \cdot \mathscr{J}_{y}=\mathscr{J}_{s y} & \text { if } \quad y^{-1} \alpha>0 \\
=\left(t_{\alpha}-1\right) \mathscr{J}_{y}+t_{\alpha} \mathscr{J}_{s y} & \text { if } \quad y^{-1} \alpha<0 \\
\left(s=s_{\alpha}, \alpha \in \Pi, y \in W\right), & \\
\mathscr{J}_{s} \cdot \theta_{\lambda}-\theta_{s(\lambda)} \cdot \mathscr{J}_{s}=\left(t_{\alpha}-1\right) \frac{\theta_{\lambda}-\theta_{s(\lambda)}}{1-\theta_{-\alpha}} & \text { if }\left\langle\alpha^{\vee}, L\right\rangle=\mathbb{Z} \\
=\left\{\left(t_{\alpha}-1\right)+\theta_{-\alpha} t_{\alpha}^{1 / 2}\left(t_{\alpha}^{\prime 1 / 2}-t_{\alpha}^{\prime 1 / 2}\right)\right\} \frac{\theta_{\lambda}-\theta_{s(\lambda)}}{1-\theta_{-\alpha}} & \text { if } \quad\left\langle\alpha^{\vee}, L\right\rangle=2 \mathbb{Z} \\
\left.\left(s=s_{\alpha}, \alpha \in \Pi, \lambda \in L\right)\right) &
\end{array}
$$

and

$$
\theta_{\lambda} \cdot \theta_{\mu}=\theta_{\lambda+\mu} \quad(\lambda, \mu \in L) .
$$

It is known that the set $\left\{\theta_{\lambda} \cdot \mathscr{J}_{w}(\lambda \in L, w \in W)\right\}$ is a basis of $H_{L}$. Since $\mathscr{J}_{w}=$ $\mathscr{J}_{s_{1}} \ldots \mathscr{J}_{s_{\ell}}$ if $w=s_{1} \ldots s_{\ell}$ is a reduced expression of $w \in W$ by $\left(1.5 \mathrm{a}^{\prime}\right)$, the correspondence $\mathscr{J}_{s} \rightarrow T_{s}(s \in S)$ and $\theta_{\lambda} \rightarrow e^{\lambda}(\lambda \in L)$ extends to an injective algebra homomorphism from $H_{L}$ to $\operatorname{End}_{\mathbb{C}}(V)$. We shall hereafter identify $H_{L}$ with its image in $\operatorname{End}_{\mathbb{C}}(V)$. Thus we have $H_{L}=\sum_{w \in W} A \cdot T_{w}$ (a free $A$-module of rank $|W|$ ). Here we write $T_{w}=T_{s_{1}} \ldots T_{s_{\ell}}$ if $w=s_{1} \ldots s_{\ell}\left(s_{i} \in S\right)$ is a reduced decomposition of $w \in W$. Note also that the action of $H_{L}$ on $V$ can be identified with the left regular action of $H_{L}$.

1.6. Let $A^{\sim}$ be the quotient field of $A$. Set $V^{\sim}=A^{\sim} \otimes_{A} V$. Then the formulas 1.3, (1.5a) and (1.5b) for $f \in A^{\sim}$ define an action of the algebra $H_{L}^{\sim}=A^{\sim} \cdot H_{L}=$ $A^{\sim} \otimes_{A} H_{L}$ on $V^{\sim}$. Define an element $I_{s}\left(s=s_{\alpha}, \alpha \in \Pi\right)$ of $H_{L}^{\sim}$ by

$$
\begin{array}{ll}
I_{s}=T_{s}-\frac{t_{\alpha}-1}{1-e^{-\alpha}} & \text { if }\left\langle\alpha^{\vee}, L\right\rangle=\mathbb{Z} ; \\
=T_{s}-\frac{\left(t_{\alpha}-1\right)+e^{-\alpha} t_{\alpha}^{1 / 2}\left(t_{\alpha}^{1 / 2}-t_{\alpha}^{\prime-1 / 2}\right)}{1-e^{-2 \alpha}} & \text { if } \quad\left\langle\alpha^{\vee}, L\right\rangle=2 \mathbb{Z} .
\end{array}
$$


Then the relation $(1.5 b)$ implies that

$$
I_{s} \cdot f=s(f) \cdot I_{s} \quad\left(f \in A^{\sim}\right) .
$$

Now we define $\mathbf{c}_{\alpha} \in A^{\sim}(\alpha \in \Delta)$ by

$$
\begin{aligned}
\mathbf{c}_{\alpha} & =\frac{1-t_{\alpha} e^{\alpha}}{1-e^{\alpha}} & \text { if } \quad\left\langle\alpha^{\vee}, L\right\rangle=\mathbb{Z} ; \\
& =\frac{\left(1+t_{\alpha}^{1 / 2} t_{\alpha}^{\prime-1 / 2} e^{\alpha}\right)\left(1-t_{\alpha}^{1 / 2} t_{\alpha}^{1 / 2} e^{\alpha}\right)}{1-e^{2 \alpha}} & \text { if } \quad\left\langle\alpha^{\vee}, L\right\rangle=2 \mathbb{Z} .
\end{aligned}
$$

Then

$$
I_{s}=T_{s}+\left(\mathbf{c}_{-\alpha}-t_{\alpha}\right) .
$$

for $\alpha=\alpha(s)$ and it can be easily seen that $I_{s}^{2}=\mathbf{c}_{\alpha} \mathbf{c}_{-\alpha}$. This element $I_{s}$ appeared (implicitly) as an intertwining operator in [Matm]. See also [K] and [L].

1.7. Now we set $J_{s}=\mathbf{c}_{-\alpha(s)}^{-1} I_{s} \in H_{L}^{\sim}$ for $s \in S$. (The results which follow are essentially the same if we choose $\mathbf{c}_{\alpha(s)}^{-1} I_{s}$ instead.) We get $J_{s}^{2}=1$. Moreover we can see that the element $J_{w} \in H_{L}^{\sim}$ for $w \in W$ given by $J_{w}=J_{s_{1}} \ldots J_{s_{\ell}}$ is independent of the choice of the (not necessarily reduced) decomposition $w=s_{1} \ldots s_{\ell}\left(s_{i} \in S\right.$ ). (This fact is essentially due to Matsumoto [Matm], see also [L].) Hence the map $J: w \mapsto J_{w}(w \in W)$ is a group isomorphism. Therefore we have obtained an action of $W_{L}$ on $V^{\sim}$ through this $J$. Note that this action is different from the one in 1.3. Since $J_{w}$ reduces to $r_{w}(w \in W)$ if $t_{\alpha}, t_{\alpha}^{\prime} \rightarrow 1(\alpha \in \Delta)$, we may regard $\left\{J_{w}(w \in W)\right\}$ as a $t$-deformation of $\left\{r_{w}(w \in W)\right\}$.

Incidentally, since $J_{w} \cdot f=w(f) \cdot J_{w}$ for $f \in A$, we see that $H_{L}^{\sim}$ is isomorphic to the algebra $A^{\sim} \otimes_{A} \mathbb{C}\left[W_{L}\right]$ (twisted tensor product) by using the following lemma proved by the induction on the length of $w \in W$.

Lemma 1.8. For any $w \in W$, we have

$$
J_{w} \in\left(\prod_{\alpha>0, w^{-1} \alpha<0} \mathbf{c}_{-\alpha}\right)^{-1} T_{w}+\sum_{y<w} A^{\sim} \cdot T_{y} .
$$

Now we show the relationship between the $W_{L}$-action (through $r$ ) and the $H_{L^{-}}$ action on $V$.

Proposition 1.9. Suppose that $w \alpha=\beta$ for $w \in W$ and $\alpha, \beta \in \Pi$. Then we have

$$
r_{w} \cdot T_{s_{\alpha}}=T_{s_{\beta}} \cdot r_{w}
$$

in $\operatorname{End}_{\mathbb{C}}(V)$.

Proof. We must prove

$$
\left(r_{w} \cdot T_{s}\right)(F)=\left(T_{s^{\prime}} \cdot r_{w}\right)(F) \quad\left(s=s_{\alpha}, s^{\prime}=s_{\beta}\right)
$$

for any $F \in V$. First we consider the case $F=h_{y}(y \in W)$. Since

$$
\begin{aligned}
& T_{s}\left(h_{y}\right)=h_{s y} \quad \text { if } \quad y^{-1}(\alpha)>0 \\
& =\left(t_{\alpha}-1\right) h_{y}+t_{\alpha} h_{s y} \quad \text { if } \quad y^{-1}(\alpha)<0,
\end{aligned}
$$


we have

$$
\begin{aligned}
\left(r_{w} \cdot T_{s}\right)\left(h_{y}\right) & = \begin{cases}h_{w s y} & \text { if } y^{-1}(\alpha)>0 \\
\left(t_{\alpha}-1\right) h_{w y}+t_{\alpha} h_{w s y} & \text { if } y^{-1}(\alpha)<0\end{cases} \\
& = \begin{cases}h_{s^{\prime} w y} & \text { if }(w y)^{-1}(\beta)>0 \\
\left(t_{\alpha}-1\right) h_{w y}+t_{\alpha} h_{s^{\prime} w y} & \text { if }(w y)^{-1}(\beta)<0 .\end{cases} \\
& =\left(T_{s^{\prime}} \cdot r_{w}\right) \cdot h_{y},
\end{aligned}
$$

noting that $y^{-1} \alpha=(w y)^{-1} \beta$ and $t_{\alpha}=t_{\beta}$. Next we put $F=f h_{y}$ for $f \in A$. Assume that $\left\langle\alpha^{\vee}, L\right\rangle=\mathbb{Z}$. Then

$$
\begin{aligned}
\left(r_{w} \cdot T_{s}\right)\left(f h_{y}\right) & =r_{w}\left(s(f) T_{s} h_{y}+\left(t_{\alpha}-1\right) \frac{f-s(f)}{1-e^{-\alpha}} h_{y}\right) \\
& =w s(f)\left(r_{w} \cdot T_{s}\right) h_{y}+\left(t_{\beta}-1\right) \frac{w(f)-w s(f)}{1-e^{-w \alpha}} h_{y} \\
& =\left(T_{s^{\prime}} \cdot r_{w}\right)\left(f h_{y}\right) .
\end{aligned}
$$

The case where $\left\langle\alpha^{\vee}, L\right\rangle=2 \mathbb{Z}$ is similar. Thus we have completed the proof of the lemma, since the elements $f h_{y}(f \in A, y \in W)$ span $V$ over $\mathbb{C}$.

By the proposition above, we easily have the following corollary, which tells us the relation between the two kinds of $W_{L}$-actions on $V^{\sim}$ (through $r$ and $J$ ).

Corollary 1.10. Under the same assumption as above, we have

$$
r_{w} \cdot J_{s_{\alpha}}=J_{s_{\beta}} \cdot r_{w}
$$

in $\operatorname{End}_{\mathbb{C}}\left(V^{\sim}\right)$.

1.11. Let $U$ be an $A$-module. We call $U$ a $\left(W_{L}, H_{L}\right)$-module if the following three conditions are satisfied:

The module $U$ has both $\mathbb{C}\left[W_{L}\right]$-and $H_{L}$-module structures .

The action of $A=\mathbb{C}[L]$ on $U$ is compatible with the above module structures .

For $\alpha, \beta \in \Pi$ with $w(\alpha)=\beta(w \in W), w \cdot T_{s_{\alpha}}=T_{s_{\beta}} \cdot w$.

Our $V$ is an example of $\left(W_{L}, H_{L}\right)$-modules by 1.9. Other examples of $\left(W_{L}, H_{L}\right)$ modules are obtained by taking subquotients of $V$ (see below).

1.12. Let $X$ and $Y$ be $\left(W_{L}, H_{L}\right)$-submodules of $V$ with $X \supset Y$. Then the subquotient of $V, X / Y$ is also a $\left(W_{L}, H_{L}\right)$-module.

We may proceed as follows to construct such $X$ and $Y$ explicitly (cf. the Lusztig-Lascoux-Schützenberger operators in [C2]). Let $U$ be the $\mathbb{C}$-vector space spanned by $h_{w}(w \in W)$. Let $H$ (resp. $\left.\mathbb{C}[W]\right)$ be the subalgebra of $\operatorname{End}_{\mathbb{C}}(V)$ generated by $T_{s}(s \in S)$ (resp. $r_{w}(w \in W)$ ). Then $H$ (resp. $\mathbb{C}[W]$ ) is isomorphic to the Hecke algebra of $W$ (resp. the group algebra of $W$ ). This $U$ has a $(W, H)$ module structure. Namely, $U$ satisfies (1.11a) (for $\mathbb{C}[W]$ and $H$ ) and (1.11c). Let $X_{0}$ and $Y_{0}$ be $(W, H)$-submodules of $U$ with $X_{0} \supset Y_{0}$. Since $A \otimes_{\mathbb{C}} X_{0}$ and $A \otimes_{\mathbb{C}} Y_{0}$ are $\left(W_{L}, H_{L}\right)$-submodules of $V$, we get the subquotient $A \otimes_{\mathbb{C}}\left(X_{0} / Y_{0}\right) \simeq$ $\left(A \otimes_{\mathbb{C}} X_{0}\right) /\left(A \otimes_{\mathbb{C}} Y_{0}\right)$ of $V$, where $X_{0} / Y_{0}$ is a $(W, H)$-subquotient of $U$.

For example, let $I$ be a subset of $S$ and $W_{I}$ the corresponding parabolic subgroup of $W ; W_{I}=\langle s(s \in I)\rangle \subset W$. Then the $\mathbb{C}$-subspace $U_{I}^{\text {triv }}$ of $U$ spanned by all the 
elements of the form $\sum_{z \in W_{I}} h_{y z}(y \in W)$ is a $(W, H)$-submodule of $U$. Note that as $W$-modules, $U_{I}^{\text {triv }} \simeq \operatorname{Ind}_{W_{I}}^{W}(1)$, the $W$-module induced from the trivial representation of $W_{I}$.

Similarly, for $I \subset S$ above, we define $U_{I}^{\text {sgn }}$ to be the $\mathbb{C}$-subspace of $U$ spanned by all the elements of the form $h_{w}+h_{w s}(w \in W, s \in I)$. Then we can easily see that this $U_{I}^{\mathrm{sgn}}$ is a $(W, H)$-submodule of $U$, and obtain a $(W, H)$-module $U / U_{I}^{\mathrm{sgn}}$. Note that as $W$-modules, $U / U_{I}^{\text {sgn }} \simeq \operatorname{Ind}_{W_{I}}^{W}(\operatorname{sgn})$, the $W$-module induced from the sign representation of $W_{I}$.

Generally, by combining the above two ways, we can produce many $\left(W_{L}, H_{L}\right)$ modules. Especially in the case where $\Delta$ is of type $A$, we can have $\left(W_{L}, H_{L}\right)$-modules for each irreducible representation of $W$ (or of $H$ ).

\section{R-Matrix Associated with Root Systems}

2.1. Now we define the R-matrix as follows. We set

$$
R_{\alpha}=r_{s_{\alpha}} \cdot J_{s_{\alpha}} \in \operatorname{End}_{\mathbb{C}}\left(V^{\sim}\right)
$$

for $\alpha \in \Pi$. Note that $R_{\alpha}$ is actually in $\operatorname{End}_{A^{\sim}}\left(V^{\sim}\right)$. Namely, $R_{\alpha}$ can be represented by a $|W| \times|W|$ matrix with coefficients in $A^{\sim}$. More generally we define $R_{\beta} \in$ $\operatorname{End}_{A^{\sim}} \sim\left(V^{\sim}\right)$ for an arbitrary root $\beta \in \Delta$ by

$$
R_{\beta}=r_{w} \cdot R_{\alpha} \cdot r_{w}^{-1}
$$

for $w \in W$ and $\alpha \in \Pi$ with $w(\alpha)=\beta$. The definition above is independent of the choice of $w$ and $\alpha$. Indeed, if $y\left(\alpha^{\prime}\right)=\beta$ for $y \in W$ and $\alpha^{\prime} \in \Pi$, then $\left(y^{-1} w\right) \alpha=\alpha^{\prime}$. This implies $r_{w} \cdot R_{\alpha} \cdot r_{w}^{-1}=r_{y} \cdot R_{\alpha^{\prime}} \cdot r_{y}^{-1}$ by 1.10 .

From the definition, we have $R_{-\alpha}=J_{s_{\alpha}} \cdot r_{s_{\alpha}}=R_{\alpha}^{-1}$ for $\alpha \in \Pi$. This shows the next proposition.

Proposition 2.2. $R_{-\beta}=R_{\beta}^{-1}$ for any $\beta \in \Delta$.

2.3. We have the formula

$$
\begin{aligned}
R_{\alpha} h_{y} & =\mathbf{c}_{\alpha}^{-1} h_{y}+\left(1-t_{\alpha} \mathbf{c}_{\alpha}^{-1}\right) h_{s y_{\alpha}} & \text { if } & y^{-1} \alpha>0 \\
& =t_{\alpha} \mathbf{c}_{\alpha}^{-1} h_{y}+\left(1-\mathbf{c}_{\alpha}^{-1}\right) h_{s y_{\alpha}} & \text { if } & y^{-1} \alpha<0
\end{aligned}
$$

for $\alpha \in \Delta$ and $y \in W$. (If $\alpha \in \Pi$, this formula is deduced immediately from (1.6.1), 1.7 , and the definition of $R_{\alpha}$. The general case follows from this.)

Theorem 2.4. Our R-matrix $\left\{R_{\alpha}(\alpha \in \Delta)\right\}$ satisfies the following Yang-Baxter equations for each rank 2 root subsystem $\Delta^{\prime}$ of $\Delta$ with simple roots $\alpha$ and $\beta$, where $\alpha$ is long and $\beta$ is short if $|\alpha| \neq|\beta|$ :

$$
\begin{aligned}
A_{1} \times A_{1} & : R_{\alpha} R_{\beta}=R_{\alpha} R_{\beta}, \\
A_{2} & : R_{\alpha} R_{\alpha+\beta} R_{\beta}=R_{\beta} R_{\alpha+\beta} R_{\alpha}, \\
B_{2} & : R_{\alpha} R_{\alpha+\beta} R_{\alpha+2 \beta} R_{\beta}=R_{\beta} R_{\alpha+2 \beta} R_{\alpha+\beta} R_{\alpha}, \\
G_{2} & : R_{\alpha} R_{\alpha+\beta} R_{2 \alpha+3 \beta} R_{\alpha+2 \beta} R_{\alpha+3 \beta} R_{\beta}=R_{\beta} R_{\alpha+3 \beta} R_{\alpha+2 \beta} R_{2 \alpha+3 \beta} R_{\alpha+\beta} R_{\alpha},
\end{aligned}
$$

respectively in the case where $\Delta^{\prime}$ is of type $A_{1} \times A_{1}, A_{2}, B_{2}$ or $G_{2}$. 
Proof. In the case $A_{1} \times A_{1}$, the statement follows from (2.3.1) and the fact $y^{-1} \alpha=$ $\left(s_{\beta} y\right)^{-1} \alpha$. Now we consider the other cases. Fix $z \in W$. Since $R_{\alpha}, R_{\beta}, R_{\alpha+\beta}, \ldots$ stabilize the $A^{\sim}$-submodule of $V^{\sim}$ spanned by $h_{y z}, y \in W\left(\Delta^{\prime}\right)$ (the Weyl group of $\left.\Delta^{\prime}\right),(2.3 .1)$ shows that we can reduce the problem to the case $\Delta^{\prime}=\Delta$. Assume, for example, that $\Delta$ is of type $A_{2}$. Then we see that

$$
\begin{aligned}
R_{\alpha} R_{\alpha+\beta} R_{\beta} & =\left(r_{s^{\prime}} r_{s} r_{s^{\prime}} J_{s^{\prime}} r_{s^{\prime}} r_{s^{\prime}}\right)\left(r_{s^{\prime}} r_{s} J_{s^{\prime}} r_{s^{\prime}}\right)\left(r_{s^{\prime}} J_{s^{\prime}}\right) \\
& =r_{s^{\prime}} r_{s} r_{s^{\prime}} J_{s^{\prime}} J_{s} J_{s^{\prime}}=r_{w_{0}} J_{w_{0}},
\end{aligned}
$$

where $s=s_{\alpha}, s^{\prime}=s_{\beta}$, and $w_{0}$ is the longest element in $W\left(A_{2}\right)$. Similarly we get

$$
R_{\beta} R_{\alpha+\beta} R_{\alpha}=r_{s} r_{s^{\prime}} r_{s} J_{s} J_{s^{\prime}} J_{s}=r_{w_{0}} J_{w_{0}} .
$$

Other cases also follow from the fact that there are two ways for giving the reduced expression of the longest element of the Weyl group of rank 2 root systems.

We remark here that besides 1.10 only the braid relations (e.g., $r_{s} r_{s^{\prime}} r_{s}=r_{s^{\prime}} r_{s} r_{s^{\prime}}$ and $\left.J_{s} J_{s^{\prime}} J_{s}=J_{s^{\prime}} J_{s} J_{s^{\prime}}\right)$ are sufficient to establish the Yang-Baxter equations in the argument above. (The conditions $r_{s}^{2}=J_{s}^{2}=1$ are not essential here.) Therefore we can state the theorem in a more abstract way (cf. 2.7 below).

It should be noted that if the root system is of type $A$, our R-matrix given above is strongly connected with the one in $[\mathrm{J}]$ produced from a Hecke algebra (See also [C2] for other types; our $I_{s}$ also appeared there in connection with the R-matrix.) But the use of the $W$-action through $r$ here seems to be new, although some $W$-actions are incorporated in the study of the R-matrix in [C2, C4]. See also Subsect. 2.6 below. Theorem 2.4 together with Proposition 2.2 reads as follows: Our $\left\{R_{\alpha}(\alpha \in \Delta)\right\}$ is a closed, $W$-invariant quantum R-matrix in the sense of Cherednik [C4].

Remark 2.5. For $w \in W$, we set $R_{w}=r_{w}^{-1} \cdot J_{w} \in \operatorname{End}_{A} \sim\left(V^{\sim}\right)$. Then for any decomposition of $w, w=s_{1} \ldots s_{\ell}$ (not necessary reduced) with $s_{k} \in S, \alpha\left(s_{k}\right)=\alpha_{k} \in \Pi$, we see that

$$
\begin{aligned}
R_{w} & =\left(r_{s_{\ell} \ldots s_{2}} R_{\alpha_{1}} r_{s_{\ell} \ldots s_{2}}^{-1}\right)\left(r_{s_{\ell} \ldots s_{3}} R_{\alpha_{2}} r_{s_{\ell} \ldots s_{3}}^{-1}\right) \ldots\left(r_{s_{\ell}} R_{\alpha_{\ell-1}} r_{s_{\ell}}^{-1}\right) R_{\alpha_{\ell}} \\
& =R_{\beta_{1}} R_{\beta_{2}} \ldots R_{\beta_{\ell}} \quad\left(\beta_{k}=s_{\ell} \ldots s_{k+1}\left(\alpha_{k}\right) \text { for } 1 \leqq k \leqq \ell\right)
\end{aligned}
$$

Note that $w^{-1}=s_{\beta_{1}} \ldots s_{\beta_{\ell}}$ and that $\ell\left(s_{\beta_{i}} \ldots s_{\beta_{\ell}}\right)-\ell\left(s_{\beta_{i+\ell}} \ldots s_{\beta_{\ell}}\right)=1($ resp. -1$)$ if $\beta_{i}>0$ (resp. $\beta_{i}<0$ ). By definition, we have

$$
R_{y z}=\left(r_{z^{-1}} \cdot R_{y} \cdot r_{z}\right) R_{z}
$$

for any $y, z \in W$ (cf. [C1]).

2.6. The discussion given above shows that we can get the R-matrix on $A^{\sim} \otimes_{A} U$ if $U$ is a $\left(W_{L}, H_{L}\right)$-module. Particularly, we have the R-matrix for each $\left(W_{L}, H_{L}\right)$ subquotient of $V$. (See 1.12 for the construction of these subquotients.) Set $V_{0}=A \otimes_{\mathbb{C}} U_{S}^{\text {triv }}(\simeq A)$ (see 1.12). This is a $\left(W_{L}, H_{L}\right)$-module. But if we regard this $V_{0}$ as a $(W, H)$-module, then we get $\left(W_{L}, H_{L}\right)$-module $V=A \otimes_{\mathbb{C}} V_{0}$. Therefore we have an R-matrix on $V^{\sim}=A^{\sim} \otimes_{\mathbb{C}} V_{0}$, the one which appeared in Cherednik [C4; Proposition 3.8]. Thus his R-matrix is essentially the same as ours in 2.1. But we think that our formulation given in this paper makes the meaning of the Yang-Baxter equations clearer. 
2.7. We can define some variant of our R-matrix. Set

$$
R_{\alpha}^{+}=r_{s_{\alpha}} \cdot T_{s_{\alpha}} \in \operatorname{End}_{\mathbb{C}}(V)
$$

for $\alpha \in \Pi$ and put $R_{\beta}^{+}=r_{w} \cdot R_{\alpha}^{+} \cdot r_{w}^{-1}$ for arbitrary $\beta \in \Delta$ by using $w \in W$ and $\alpha \in \Pi$ with $w \alpha=\beta$ as in 2.1. By 1.9 , we see that this $R$-matrix $\left\{R_{\alpha}^{+}(\alpha \in \Delta)\right\}$ is well-defined and satisfies the YBE as in 2.4. Note that $R_{\alpha}^{+}$is neither $A$-linear nor satisfying 2.2 in this case. The subquotients of this R-matrix for the 1-dimensional representations of $W$ appear in [C2].

\section{R-Matrix Associated with Affine Root Systems}

In this section, we extend our R-matrix to affine root systems following Cherednik [C4]. By using this extension, we define the quantum Knizhnik-Zamolodchikov equations. The basic idea and formalism employed here are due to Cherednik. But we include some of the arguments in [C4] here for our later use.

3.1. Let $M$ be a $W$-invariant lattice in $E$. Then for each $\alpha \in \Delta$, we have a lattice $\langle\alpha, M\rangle$ in $\mathbb{R}$. Obviously $\langle\alpha, M\rangle=\langle\beta, M\rangle$ if $\alpha$ and $\beta$ are $W$-conjugate. For $\alpha \in \Delta$ and $m \in\langle\alpha, M\rangle$, let $\alpha+m$ be the affine function on $E$ defined to be $(\alpha+m)(x)=$ $\langle x, \alpha\rangle+m(x \in E)$. We put

$$
\Delta^{\sim}=\Delta(M)^{\sim}=\{\alpha+m ; \alpha \in \Delta, m \in\langle\alpha, M\rangle\}
$$

and call this the affine root system corresponding to $\Delta$ and $M$ (cf. [Macl]). The affine root system $\Delta^{\sim}$ contains $\Delta$ in an obvious manner. Let $H_{\alpha+m}$ be the hyperplane in $E$ given by

$$
H_{\alpha+m}=\{x \in E ;(\alpha+m)(x)=0\}
$$

for $\alpha+m \in \Delta^{\sim}$. The set of these hyperplanes are invariant under the action of $W$ on $E ; w\left(H_{\alpha+m}\right)=H_{w \alpha+m}$ for $w \in W$. We define the action of $W$ on $\Delta^{\sim}$ by $w(\alpha+m)=w \alpha+m$. Define the translation action of $M$ on $E$ by $\tau_{\mu}(x)=x+\mu(x \in$ $E, \mu \in M)$. We easily see that $\tau_{\mu}\left(H_{\alpha+m}\right)=H_{\alpha+(m-\langle\mu, \alpha\rangle)}$, We define the action of $M$ on $\Delta^{\sim}$ by $\tau_{\mu}(\alpha+m)=\alpha+(m-\langle\mu, \alpha\rangle)$.

Let $W_{M}$ be the subgroup of the affine transformation group of $E$ defined to be the semidirect product of $M$ and $W$. This $W_{M}$ acts on $\Delta^{\sim}$, since both $W$ and $M$ act on $\Delta^{\sim}$ compatibly. It is known that $W_{M}$ acts, as an affine transformation group, transitively on alcoves, i.e., the set of connected components of $E \backslash \bigcup_{\alpha+m \in \Delta^{\sim}} H_{\alpha+m}$, and that

$$
C=\left\{x \in E ; 0<\langle x, \alpha\rangle<n_{\alpha}\left(\alpha \in \Delta^{+}\right)\right\}
$$

is an alcove, where $n_{\alpha}$ is the generator of $\langle\alpha, M\rangle$ with $n_{\alpha}>0$. We call an affine root $\alpha+m$ positive if $(\alpha+m)(C)>0$. Note that all elements of $\Delta^{+}$are positive, and that either $\alpha+m$ or $-\alpha-m$ is positive.

3.2. For $w \in W_{M}$, we can choose a sequence of alcoves

$$
w^{-1} C=C_{0}, C_{1}, \ldots, C_{\ell}=C,
$$

so that there is only one hyperplane $H_{\alpha_{i}+m_{i}}$ separating $C_{i-1}$ and $C_{i}$. We assume that $\left(\alpha_{i}+m_{i}\right)\left(C_{i-1}\right)<0$ and $\left(\alpha_{i}+m_{i}\right)\left(C_{i}\right)>0$. We call these ordered affine roots, 
$\alpha_{1}+m_{1}, \ldots, \alpha_{\ell}+m_{\ell}$, a root sequence from $w^{-1} C$ (to $C$ ). If we denote by $s_{\alpha_{i}+m_{l}} \in$ $W_{M}$ the reflection with respect to the hyperplane $H_{\alpha_{i}+m_{i}}$, then we have

$$
C_{i}=s_{\alpha_{i}+m_{i}} \ldots s_{\alpha_{\ell}+m_{\ell}} C(1 \leqq i \leqq \ell) \text {. }
$$

Especially

$$
w^{-1} C=s_{\alpha_{1}+m_{1}} \ldots s_{\alpha_{\ell}+m_{\ell}} C
$$

(cf. 2.5). Let $\Omega$ be the stabilizer of $C$ in $W_{M}$. The equality above implies that $w^{-1}=s_{\alpha_{1}+m_{1}} \ldots s_{\alpha_{\ell}+m_{\ell}} \gamma$ for some $\gamma \in \Omega$.

3.3. Now we extend our R-matrix in Sect. 2, defined for $\alpha \in \Delta$ to each affine root $\alpha+m \in \Delta^{\sim}$. by

We fix $q$, a real positive number. Let $L_{x}$ be the $q$-shift operator on $A^{\sim}$ defined

$$
L_{x}\left(e^{\lambda}\right)=q^{\langle x, \lambda\rangle} e^{\lambda}
$$

for $x \in E$ and $\lambda \in L$. We regard this $L_{x}$ as also acting on $V^{\sim}$ by

$$
L_{x}\left(\sum f_{w} h_{w}\right)=\sum L_{x}\left(f_{w}\right) h_{w} .
$$

Note that $L_{-x}=L_{x}^{-1}$ and $r_{w} \cdot L_{x} \cdot r_{w}^{-1}=L_{w x}$ for $w \in W$.

Next we denote by $P_{x}^{u}(u, x \in E)$ the element of $\operatorname{End}_{A} \sim\left(V^{\sim}\right)$ given by

$$
P_{x}^{u}\left(h_{w}\right)=q^{\langle x, w u\rangle} h_{w}(w \in W),
$$

cf. [FR]. Note that $P_{-x}^{u}=\left(P_{x}^{u}\right)^{-1}=P_{x}^{-u}$ and $r_{w} \cdot P_{x}^{u} \cdot r_{w}^{-1}=P_{w x}^{u}$ for $w \in W$.

The operators $L_{x}(x \in E)$ and $P_{x}^{u}(u, x \in E)$ commute each other.

Lemma 3.4. If $\langle x, \alpha\rangle=0$ for $x \in E$ and $\alpha \in \Delta$, then the both $L_{x}$ and $P_{x}^{u}(u$ arbitrary) commute with $R_{\alpha}$.

This is clear from 2.2 .

3.5. Now we fix $u \in E$ hereafter and put $P_{x}=P_{x}^{u}$ for simplicity. Set $r_{x}=P_{x} L_{-x} \in$ $\operatorname{End}_{\mathbb{C}}\left(V^{\sim}\right)$. We note that $r_{w}(w \in W)$ in 1.4 and these $r_{\mu}(\mu \in M)$ generate a subgroup of $\operatorname{Aut}_{\mathbb{C}}\left(V^{\sim}\right)$ naturally isomorphic to $W_{M}$. Since $r_{w} \cdot e^{\lambda} \cdot r_{w}^{-1}=e^{w \lambda}(w \in W)$ and $r_{\mu} \cdot e^{\lambda} \cdot r_{\mu}^{-1}=q^{\langle\mu, \lambda\rangle} e^{\lambda}(\mu \in M)$ in $\operatorname{End}_{\mathbb{C}}\left(V^{\sim}\right)$ for $\lambda \in L, W_{M}$ stabilizes $A^{\sim} \subset$ $\operatorname{End}_{\mathbb{C}}\left(V^{\sim}\right)$ under this action. For an affine root $\alpha+m \in \Delta^{\sim}$, we set

$$
R_{\alpha+m}=r_{x}^{-1} \cdot R_{\alpha} \cdot r_{x},
$$

provided that $x \in E$ satisfies $\langle x, \alpha\rangle=m$. This $R_{\alpha+m}$ is well defined thanks to 3.4 . Thus if we denote by $r_{w}$ for $w \in W_{M}$ the corresponding element in $\operatorname{Aut}_{\mathbb{C}}\left(V^{\sim}\right)$ above, we have

$$
R_{w(\alpha+m)}=r_{w} \cdot R_{\alpha+m} \cdot r_{w}^{-1}
$$

(cf. 2.1). (Note that $r_{\tau_{\mu}}=r_{\mu}$ for $\mu \in M$ ). Incidentally we have the explicit formula of $R_{\alpha+m}$ from (2.3.1) as follows:

$$
\begin{aligned}
& R_{\alpha+m} h_{y}=\mathbf{c}_{\alpha+m}^{-1} h_{y}+q^{m\left\langle\alpha^{\vee}, y u\right\rangle}\left(1-t_{\alpha} \mathbf{c}_{\alpha+m}^{-1}\right) h_{s_{\alpha} y} \quad \text { if } \quad y^{-1} \alpha>0 \\
& =t_{\alpha} \mathbf{c}_{\alpha+m}^{-1} h_{y}+q^{m\left\langle\alpha^{\vee}, y u\right\rangle}\left(1-\mathbf{c}_{\alpha+m}^{-1}\right) h_{s_{\alpha} y} \quad \text { if } \quad y^{-1} \alpha<0 .
\end{aligned}
$$


Here we put $\mathbf{c}_{\alpha+m}=L_{x}\left(\mathbf{c}_{\alpha}\right),\langle\alpha, x\rangle=m$. (Since $r_{x}^{-1} \cdot e^{\alpha} \cdot r_{x}=L_{x}\left(e^{\alpha}\right)=q^{m} e^{\alpha}, \mathbf{c}_{\alpha+m}$ is obtained from $\mathbf{c}_{\alpha}$ by replacing $e^{\alpha}$ in (1.6.1) by $q^{m} e^{\alpha}$.)

Proposition 3.6. For $\alpha+m, \beta+n \in \Delta^{\sim}$, we suppose that $\alpha, \beta \in \Delta$ satisfy the condition as in Theorem 2.4. Then we have the same formulas of type $A_{1} \times A_{1}, A_{2}, B_{2}$ or $G_{2}$ in Theorem 2.4, if we replace $\alpha, \beta \in \Delta$ by $\alpha+m, \beta+n$ respectively.

This is obvious, because there is an element $x \in E$ satisfying $\langle x, \alpha\rangle=m$ and $\langle x, \beta\rangle=n$.

\subsection{We put}

$$
R_{w}=R_{\alpha_{1}+m_{1}} \ldots R_{\alpha_{\ell}+m_{\ell}} \in \operatorname{End}_{A} \sim\left(V^{\sim}\right)
$$

for $w \in W_{M}$ (cf. 2.5). Here we choose a root sequence from $w^{-1} C, \alpha_{1}+m_{1}, \ldots, \alpha_{\ell}+$ $m_{\ell} \in \Delta^{\sim}$ as in 3.1. Proposition 3.6 and the property $R_{-\alpha-m}=R_{\alpha+m}^{-1}$ (cf. 2.2) show that this $R_{w}$ is independent of the choice of the root sequence $\alpha_{1}+m_{1}, \ldots, \alpha_{\ell}+m_{\ell}$ above. We note that, in the affine case we still have the following relation.

Proposition 3.8. We have

$$
R_{y w}=\left(r_{w}^{-1} \cdot R_{y} \cdot r_{w}\right) R_{w}
$$

for any $w, y \in W_{M}$.

Proof. We see easily that $w^{-1}\left(\beta_{1}+n_{1}\right), \ldots, w^{-1}\left(\beta_{r}+n_{r}\right), \alpha_{1}+m_{1}, \ldots, \alpha_{\ell}+m_{\ell}$ is a root sequence from $(y w)^{-1} C$ if $\alpha_{1}+m_{1}, \ldots, \alpha_{\ell}+m_{\ell}$ (resp. $\beta_{1}+n_{1}, \ldots, \beta_{r}+n_{r}$ ) is a one from $w^{-1} C$ (resp. $\left.y^{-1} C\right)$.

Proposition 3.9. Set $J_{w}=r_{w} \cdot R_{w}$ for $w \in W_{M}$. Then we have $J_{y} J_{w}=$ $J_{y w}\left(y, w \in W_{M}\right)$.

Proof. This is obvious from 3.8 above, since

$$
J_{y} J_{w}=\left(r_{y} \cdot R_{y}\right)\left(r_{w} \cdot R_{w}\right)=r_{y w}\left(r_{w}^{-1} \cdot R_{y} \cdot r_{w}\right) R_{w}=r_{y w} \cdot R_{y w}=J_{y w} .
$$

Remark 3.10. Define a group structure on $H(E, E)=E \times E \times \mathbb{R}$ by

$$
(x, u, a) \cdot\left(x^{\prime}, u^{\prime}, a^{\prime}\right)=\left(x+x^{\prime}, u+u^{\prime}, a+a^{\prime}+\left\langle u, x^{\prime}\right\rangle-\left\langle x, u^{\prime}\right\rangle\right),
$$

where $x, x^{\prime}, u, u^{\prime} \in E$ and $a, a^{\prime} \in \mathbb{R}$. This is a Heisenberg group. We denote by $H(L, M)$ the subgroup of $H(E, E)$ generated by $L \times\{0\} \times\{0\} \simeq L$ and $\{0\} \times M \times\{0\} \simeq M$. Since $W$ acts on $H(E, E)$ as a group automorphism by $w(x, u, a)=(w x, w u, a)$, and stabilizes $H(L, M)$, we can define the semidirect product $W_{L, M}=W \ltimes H(L, M)$. We call this group the Heisenberg-Weyl group (for $W$-invariant lattices $L$ and $M$ ). Then it is easily seen that both of the following two subgroups of $\operatorname{Aut}_{\mathbb{C}}\left(V^{\sim}\right)$ containing $\left\langle e^{\lambda}(\lambda \in L)\right\rangle \simeq L,\left\langle e^{\lambda}(\lambda \in L), r_{w}\left(w \in W_{M}\right)\right\rangle$ and $\left\langle e^{\lambda}(\lambda \in L), J_{w}\left(w \in W_{M}\right)\right\rangle$ are naturally isomorphic to this Heisenberg-Weyl group $W_{L, M}$. We may regard the latter as a $t$-deformation of the former.

Now we have come to the position to define quantum Knizhnik-Zamolodchikov equations by using our R-matrix after Cherednik [C4] (cf. [FR]). We may replace the coefficient of $V^{\sim}\left(A^{\sim}\right.$ in this case $)$ by larger ones. Let $A^{*}$ be a commutative $A$-algebra with $W_{M}$-action that is compatible on $A$ (see 3.5). We assume that $\mathbf{c}_{\alpha+m}^{ \pm 1}$ belongs to $A^{*}$ for each $\alpha+m \in \Delta^{\sim}$. (Of course we may take $A^{\sim}$, for example.) Then we can work with our R-matrix, etc. on $V^{*}=A^{*} \otimes_{A} V$ instead of $V^{\sim}=A^{\sim} \otimes_{A} V$. 
Definition 3.11. The quantum Knizhnik-Zamolodchikov equation (QKZ) of type $u \in E$ is a system of linear q-difference equations on $V^{*}$ given by

$$
L_{\mu} F=P_{\mu}^{u} \cdot R_{\tau_{\mu}} F \quad(\forall \mu \in M) .
$$

In other words,

$$
J_{\tau_{\mu}} F=F(\forall \mu \in M) .
$$

This system is a $q$-analogue of the system of differential equations given by Cherednik and Matsuo [C2, C3, Mato]. In fact, we recover their system (in Matsuo's form) if we set $t_{\alpha}=q^{k_{\alpha}}, t_{\alpha}^{\prime}=q^{k_{\alpha}^{\prime}}\left(k_{\alpha}, k_{\alpha}^{\prime} \in \mathbb{R}\right)$ and let $q \rightarrow 1$. We note that this system of difference equations is also obtained by Cherednik [C5] in somewhat different formulations (cf. 3.13 below).

3.12. After [C4], we see that Proposition 3.9 implies the following two facts about our QKZ.

The compatibility condition of $\mathrm{QKZ}$ : This condition is equivalent to the commutativity of $J_{t_{\mu}}$ and $J_{t_{v}}$ for any $\mu, v \in M$.

The $W$-action on the solution space of $\mathrm{QKZ}$ : If $F \in V^{*}$ is a solution of QKZ, then $J_{w} F$ is also a solution since $J_{t_{\mu}} J_{w} F=J_{w} J_{t_{w}-1_{\mu}} F=J_{w} F$.

3.13. We remark that we can get a $\mathrm{QKZ}$ on $U^{*}=A^{*} \otimes_{A} U$ for a $\left(W_{L}, H_{L}\right)$-module $U$. But in this case, we may have a restriction on $u \in E$ (a parameter used in $\left.P_{x}=P_{x}^{u}\right)$. For example, if we take $U=U_{I}^{\text {triv }}$ in 1.12 , we are forced to set $z u=u$ for all $z \in W_{I}$. Actually, we can formulate a $\mathrm{QKZ}$ on $A^{*} \otimes_{A} \mathbb{C}\left[W_{L, M}\right]$, where $\mathbb{C}\left[W_{L, M}\right]$ is the group algebra of $W_{L, M}$ (note that $\mathbb{C}\left[W_{L, M}\right] \supset A=\mathbb{C}[L]$ ). This equation coincides with the one in [C5]. Then we see that our QKZ in 3.11 is given on the induced module $A^{*} \otimes_{A} \mathbb{C}\left[W_{L, M}\right] \otimes_{\mathbb{C}[M]} \mathbb{C}_{u}$, where $\mathbb{C}_{u}$ is a 1-dimensional representation of $M$ defined by $\mu \rightarrow q^{\langle\mu, u\rangle}$. This explains why the restriction above on $u \in E$ is necessary.

Incidentally, Cherednik used a specialization of the $\mathrm{QKZ}$ above (i.e. his $\mathrm{QKZ}$ $[\mathrm{C} 5 ; 3.9])$ under certain induced representations of the affine Hecke algebra $\left(\mathscr{H}_{Y}\right.$ in [C5]), so as to apply to eigenvalue problems. It is easily seen that our Eq. 3.11 is also a specialization under another kind of induced representations of the Hecke algebra $\mathscr{H}_{Y}$, principal series representations. The relation between these two kinds of representations is given by the "Poisson integrals". (See [K2] for the counterpart in the case of $p$-adic groups. The general affine Hecke algebra case can be handled similarly, cf. [K3].) Especially, for generic parameters, these two kinds of representations are isomorphic.

\section{Macdonald's Difference Operators}

Macdonald [Mac3] introduced some difference operators with coefficients in $A^{\sim}$, invariant under the action of $W$, in order to show the existence of certain symmetric polynomials. (See 4.2 below.) We show a relation between these operators and our QKZ (3.11).

In the rest of this paper, we assume that the root system $\Delta$ is irreducible.

4.1. Let $M$ be the $W$-invariant lattice in 3.1 . Set

$$
M^{*}=\{\mu \in E ;\langle\mu, \alpha\rangle \in\langle\alpha, M\rangle \text { for any } \alpha \in \Delta\} .
$$


Then $M^{*}$ is also a $W$-invariant lattice of $E$. By the irreducibility of $\Delta$, it can be easily seen that this $M^{*}$ is similar to either

or

(a) the weight lattice $P$;

(b) The coweight lattice $P^{\vee}$.

Note that $M^{*} \supset M$ and $\left\langle\alpha, M^{*}\right\rangle=\langle\alpha, M\rangle$ for any $\alpha \in \Delta$. Hence the affine root system corresponding to $\Delta$ and $M^{*}$ is the same as $\Delta^{\sim}$.

From now on, we shall assume that $M=M^{*}$, and that $M^{*}$ is normalized so that $M=\left(2|\beta|^{-2}\right) P(\beta$ is a short root of $\Delta)$ in the case (a) or $M=P^{\vee}$ in the case (b). Then $n_{\alpha}$, the positive generator of $\langle\alpha, M\rangle$ (see 3.1), is given by

or

(a) $n_{\alpha}=|\alpha|^{2} /|\beta|^{2}$

(b) $n_{\alpha}=1$

respectively (cf. [Mac3]). Following Macdonald [Mac3], we use the notation

$$
\alpha_{*}=n_{\alpha}^{-1} \cdot \alpha \quad(\alpha \in \Delta) .
$$

Thus the set $\Delta_{*}=\left\{\alpha_{*} \in E \mid \alpha \in \Delta\right\}$ is also a root system in $E$, which has the same Weyl group $W$ as $\Delta$, and $M^{*}$ is identical to the coweight lattice $P_{*}^{\vee}$ of $\Delta_{*}$. We also set

$$
q_{\alpha}=q^{n_{\alpha}} \quad(\alpha \in \Delta)
$$

Therefore we have

$$
L_{\mu}\left(e^{\alpha}\right)=q_{\alpha}^{\left\langle\mu, \alpha_{*}\right\rangle} e^{\alpha} \quad(\mu \in M, \alpha \in \Delta) .
$$

4.2. Now we give Macdonald's difference operators. For a dominant coweight $\pi \in M=P_{*}^{\vee}$, we let $W_{\pi}$ be the stabilizer of $\pi$ in $W$, and $W^{\pi}$ the set of coset representatives for $W / W_{\pi}$ consisting of the elements with minimal length. Recall that the c-functions for affine roots are defined by the formula

$$
\boldsymbol{c}_{\alpha+m}=L_{\mu}\left(\mathbf{c}_{\alpha}\right) \quad\left(\alpha+m \in \Delta^{\sim}, \mu \in M,\langle\mu, \alpha\rangle=m\right) .
$$

We treat the following two cases separately:

(a) Assume that the root system $\Delta_{*}$ admits a minuscule coweight $\pi$. Namely $\Delta$ is (hence $\Delta_{*}$ is also) a root system of type $A, B, C, D, E_{6}$ or $E_{7}$ and $\pi$ is a fundamental coweight of $\Delta_{*}$ with the condition $\left\langle\pi, \alpha_{*}\right\rangle=0$ or $1(\alpha \in \Pi)$. Then the operator $D_{\pi}$ is given by

$$
D_{\pi}=\sum_{w \in W^{\pi}}\left(\prod_{\alpha>0,\left\langle\pi, \alpha_{*}\right\rangle=1} \mathbf{c}_{w \alpha}\right) L_{w \pi} .
$$

Generally, there may be several choices for minuscule $\pi$, hence several operators $D_{\pi}$ arise.

(b) Assume that the root system $\Delta$ is of type $E_{8}, F_{4}$ or $G_{2}$. Then we let $\pi$ be the short dominant coroot of $\Delta_{*}$ and put

$$
D_{\pi}=\sum_{w \in W^{\pi}}\left(\mathbf{c}_{w \alpha_{0}} \mathbf{c}_{w \alpha_{0}+n_{\alpha_{0}}} \prod_{\alpha>0,\left\langle\pi, \alpha_{*}\right\rangle=1} \mathbf{c}_{w \alpha}\right)\left(L_{w \pi}-1\right) .
$$


Here $\left(\alpha_{0}\right)_{*}$ is the maximal root of $\Delta_{*}\left(\alpha_{0} \in \Delta\right)$ so that $\pi=\left(\alpha_{0}\right)_{*}^{\vee}=2\left(\alpha_{0}\right)_{*} /\left|\left(\alpha_{0}\right)_{*}\right|^{2}$. Note that $\left\langle\pi, \alpha_{*}\right\rangle \leqq 2$ for $\alpha>0$ and the equality holds only when $\alpha=\alpha_{0}$.

4.3. Here we shall give some remark since the notation used in [Mac3] is slightly different from ours. Let us put

$$
\begin{array}{r}
\bar{\Delta}=\left\{a_{\alpha} \alpha \in E \mid \alpha \in \Delta, a_{\alpha}= \pm 1\left(\left\langle\alpha^{\vee}, L\right\rangle=\mathbb{Z}\right) ;\right. \\
\left.a_{\alpha}= \pm 1, \pm 2\left(\left\langle\alpha^{\vee}, L\right\rangle=2 \mathbb{Z}\right)\right\} .
\end{array}
$$

This $\bar{\Delta}$ is possibly a non-reduced root system in $E$ with the same Weyl group $W$. The pair $\left(\bar{\Delta}, \Delta_{*}\right)$ is admissible in the sense of [Mac3]. For $\alpha \in \Delta$, set

$$
t_{\alpha}^{\mathrm{Mac}}=t_{\alpha} \quad\left(\left\langle\alpha^{\vee}, L\right\rangle=\mathbb{Z}\right)
$$

and

$$
t_{\alpha}^{\mathrm{Mac}}=t_{\alpha}\left(t_{\alpha}^{\prime}\right)^{-1}, \quad t_{2 \alpha}^{\mathrm{Mac}}=t_{\alpha}^{\prime} \quad\left(\left\langle\alpha^{\vee}, L\right\rangle=2 \mathbb{Z}\right) .
$$

Then we can see easily that the difference operators $E_{\pi}$ [Mac3; 5-6] (with parameters $t_{\alpha}^{\mathrm{Mac}}, \alpha \in \bar{\Delta}$ ) coincide with our $D_{\pi}$.

4.4. According to Macdonald [Mac3], the operators in 4.2 stabilize $A^{W}$, the subalgebra of $A$ consisting of $W$-invariant elements, and give an eigenspace decomposition of $A^{W}, A^{W}=\sum \mathbb{C} \cdot P_{\lambda}$ (sum over $L_{++}$, the set of dominant weights in $L$ ). The eigenfunction $P_{\lambda}\left(\lambda \in L_{++}\right)$, called the Macdonald symmetric polynomial, is characterized by the following properties (i) and (ii) (see [Mac3]):

$$
D_{\pi} P_{\lambda}=c_{\pi, \lambda} P_{\lambda}
$$

where the eigenvalue $c_{\pi, \lambda} \in \mathbb{R}$ is given by

$$
c_{\pi, \lambda}=q^{\left\langle\pi, \rho_{k}\right\rangle} \sum_{w \in W^{\pi}} q^{\left\langle w \pi, \lambda+\rho_{k}\right\rangle}
$$

or

$$
c_{\pi, \lambda}=q^{\left\langle\pi, \rho_{k}\right\rangle} \sum_{w \in W^{\pi}}\left(q^{\left\langle w \pi, \lambda+\rho_{k}\right\rangle}-q^{\left\langle w \pi, \rho_{k}\right\rangle}\right),
$$

respectively for the case $(4.2 \mathrm{a})$ or $(4.2 \mathrm{~b})$ above. Here we choose $k_{\alpha} \in \mathbb{R}$ so that $t_{\alpha}=q_{\alpha}^{k_{\alpha}}$ and set $\rho_{k}=\frac{1}{2} \sum_{\alpha>0} k_{\alpha} \alpha \in E$.

$$
P_{\lambda} \in m_{\lambda}+\sum_{\mu \in L_{++}, \mu<\lambda} \mathbb{R} \cdot m_{\mu},
$$

where $m_{\mu}=\sum_{w \in W^{\mu}} e^{w \mu}$, the monomial symmetric polynomial.

4.5. We make a comment on the eigenvalues that appear in (4.4a) and (4.4b). For $w \in W$ with a reduced decomposition $w=s_{1} \ldots s_{\ell}$, we set

$$
t_{w}=t_{\alpha\left(s_{1}\right)} \ldots t_{\alpha\left(s_{\ell}\right)} .
$$

This $t_{w}$ is well-defined (see [B]) and is equal to $\prod_{\alpha>0, w^{-1} \alpha<0} t_{\alpha}=\prod_{\alpha>0, w \alpha<0} t_{\alpha}$. Note that

$$
t_{\alpha}^{\left\langle\pi, \alpha_{*}\right\rangle}=q^{k_{\alpha}\langle\pi, \alpha\rangle} .
$$


On the other hand, since $s_{\alpha} \rho_{k}=\rho_{k}-k_{\alpha} \alpha$, we have

$$
w \rho_{k}=\rho_{k}-\sum_{\alpha>0, w^{-1} \alpha<0} k_{\alpha} \alpha .
$$

Hence we have for $w \in W$,

$$
q^{\left\langle w \pi, \rho_{k}\right\rangle+\left\langle\pi, \rho_{k}\right\rangle}=\prod_{\alpha>0, w \alpha>0} q^{k_{\alpha}\langle\pi, \alpha\rangle}=\prod_{\alpha>0, w \alpha>0} t_{\alpha}^{\left\langle\pi, \alpha_{*}\right\rangle},
$$

which is equal to $t_{w_{0} w w_{\pi}}$ in the case (4.2a) since $\left\langle\pi, \alpha_{*}\right\rangle \leqq 1$ and $w \in W^{\pi}$. Here $w_{0}$ and $w_{\pi}$ are the longest elements of $W$ and $W_{\pi}$ respectively. In the case (4.2b), similarly we have

$$
\begin{aligned}
q^{\left\langle w \pi, \rho_{k}\right\rangle+\left\langle\pi, \rho_{k}\right\rangle} & =t_{w_{0} w w_{\pi}} t_{\alpha_{0}} & \text { if } & w \alpha_{0}>0 \\
& =t_{w_{0} w w_{\pi}} & \text { if } & w \alpha_{0}<0
\end{aligned}
$$

Therefore we get

$$
c_{\pi, \lambda}=\sum_{w \in W^{\pi}} t_{w_{0} w w_{\pi}} q^{\langle w \pi, \lambda\rangle}=\sum_{w \in W^{\pi}} t_{w} q^{\left\langle w_{0} w \pi, \lambda\right\rangle}
$$

or

$$
\begin{aligned}
c_{\pi, \lambda} & =\sum_{w \in W^{\pi}, w \alpha_{0}>0}\left\{t_{w_{0} w w_{\pi}} t_{\alpha_{0}} q^{\langle w \pi, \lambda\rangle}+t_{w_{0} w s_{\alpha_{0}} w_{\pi}} q^{-\langle w \pi, \lambda\rangle}-t_{w_{0} w w_{\pi}} t_{\alpha_{0}}-t_{w_{0} w s_{0} w_{0}}\right\} \\
& =\sum_{w \in W^{\pi}, w \alpha_{0}<0}\left\{t_{w} t_{\alpha_{0}} q^{-\langle w \pi, \lambda\rangle}+t_{w \alpha_{0}} q^{\langle w \pi, \lambda\rangle}-t_{w} t_{\alpha_{0}}-t_{w s_{\alpha_{0}}}\right\},
\end{aligned}
$$

respectively in the case (4.4a) or (4.4b), since $w_{0} w w_{\pi} \in W^{\pi}$ if and only if $w \in W^{\pi}$.

We note that the formulas (4.5a) and (4.5b) above make sense even for $\lambda$ in $E$, not in $L$.

Now we can state the following theorem, which may be viewed as a $q$-analogue of a part of [Mato] (and [C3]).

Theorem 4.6 Assume $F=\sum_{w \in W} f_{w} h_{w} \in V^{*}$ to be a solution of QKZ for $u \in E$. Then the sum $F^{0}=\sum_{w \in W} t_{w} f_{w} \in A^{*}$ is an eigenfunction of Macdonald's difference operator $D_{\pi}$ with the eigenvalue $c_{\pi, w_{0} u}$.

We prove this theorem in the next section.

A similar statement can be found in [C5] for the case of type A. (In [C5], more general eigenvalue problems are considered; but explicit forms of difference operators which appear in [C5] do not seem to be known for the other types.)

\section{Proof of Theorem 4.6}

In this section we shall give a proof of Theorem 4.6. We may (and shall) assume that $A^{*}=A^{\sim}$, since all the arguments given below are formal and based on the algebraic relations like $(1.5 \mathrm{~b})$, etc. We put $H^{\sim}=H_{L}^{\sim}$ for simplicity.

5.1. First we show some preliminary lemmas on Hecke algebras. We consider

$$
\left(H^{\sim}\right)^{n}=\left\{\vec{G}=\left(G_{1}, \ldots, G_{n}\right) \mid G_{i} \in H^{\sim}(i=1, \ldots, n)\right\}
$$


as an $H^{\sim}$-bimodule under the natural left and right actions of $H^{\sim}$. The group $G L(n, \mathbb{C})$ acts on $\left(H^{\sim}\right)^{n}$ from the right-hand side. Note that this action commutes with both the left and right actions of $H^{\sim}$. Every element $\vec{G}=\left(G_{1}, \ldots, G_{n}\right)$ of $\left(H^{\sim}\right)^{n}$ can be written as

$$
\vec{G}=\sum_{w \in W} \vec{g}_{w} \cdot T_{w}
$$

for some $\vec{g}_{w}=\left(g_{1 w}, \ldots, g_{n w}\right) \in\left(A^{\sim}\right)^{n} \subset\left(H^{\sim}\right)^{n}$ (cf. 1.5).

Let $\rho: W \rightarrow G L(n, \mathbb{C})$ be a representation of $W$ and let

$$
\mathbf{c}=\prod_{\alpha>0} \mathbf{c}_{\alpha} .
$$

Lemma 5.2. An element $\vec{G} \in\left(H^{\sim}\right)^{n}$ satisfies the condition

$$
J_{w} \cdot \vec{G}=\vec{G} \cdot \rho(w) \quad(\forall w \in W)
$$

if and only if there exists $\vec{g}=\left(g_{1}, \ldots, g_{n}\right) \in\left(A^{\sim}\right)^{n}$ such that

$$
\vec{G}=\sum_{w \in W} J_{w} \mathbf{c} \cdot \vec{g} \cdot \rho(w)^{-1} .
$$

Proof. The "if" part is trivial. To prove the "only if" part, we note that $\vec{G}$ can be decomposed as $\vec{G}=\sum_{y \in W} \vec{g}_{y}^{\prime} J_{y}$ for some $\vec{g}_{y}^{\prime} \in\left(A^{\sim}\right)^{n}(w \in W)$, see 1.8. Thus

$$
\begin{aligned}
\vec{G} & =|W|^{-1} \sum_{w \in W} J_{w} \vec{G} \rho(w)^{-1} \\
& =|W|^{-1} \sum_{y, w \in W} J_{w} \vec{g}_{y}^{\prime} J_{y} \rho(w)^{-1} \\
& =\sum_{z \in W} J_{z}\left\{|W|^{-1} \sum_{y \in W} y^{-1}\left(\vec{g}_{y}^{\prime}\right) \rho(y)\right\} \rho(z)^{-1} .
\end{aligned}
$$

Here we denote by $y^{-1}\left(\vec{g}_{y}^{\prime}\right)$ the image of $\vec{g}_{y}^{\prime}$ under the natural action of $y^{-1} \in W$ on $\left(A^{\sim}\right)^{n}$. This shows the lemma.

Now we set $\chi=\sum_{w \in W} T_{w}$. Then we have $T_{s} \cdot \chi=t_{\alpha(s)} \chi$ for $s \in S$. Hence $J_{s}(g \chi)=s(g) \chi(s \in S)$, which implies $J_{w}(g \chi)=w(g) \chi$ for $g \in A^{\sim}$.

Lemma 5.3. Suppose that $\vec{G}=\sum_{w \in W} \vec{g}_{w} \cdot T_{w} \in\left(H^{\sim}\right)^{n}$ satisfies the condition (5.2.1) above. Then we have

$$
\vec{G} \cdot \chi=\sum_{z \in W} z(\mathbf{c})\left(z w_{0}\right)\left(\vec{g}_{w}\right) \chi \cdot \rho\left(z w_{0}\right)^{-1}
$$

for any $w \in W$.

Proof. We proceed by downward induction on the length $\ell(w)$. First we consider the case $w=w_{0}$. Since

$$
J_{w_{0}} \cdot \mathbf{c} \in T_{w_{0}}+\sum_{y<w_{0}} A^{\sim} \cdot T_{y}
$$


and

$$
J_{y} \cdot \mathbf{c} \in \sum_{y<w_{0}} A^{\sim} \cdot T_{y}\left(y<w_{0}\right)
$$

by 1.10 , we see that

$$
\vec{g}_{w_{0}}=w_{0}(\vec{g}) \cdot \rho\left(w_{0}\right)^{-1}
$$

from the expression (5.2.2). Therefore

$$
\begin{aligned}
\vec{G} \cdot \chi & =\sum_{z \in W} J_{z} \mathbf{c} w_{0}\left(\vec{g}_{w_{0}}\right) \cdot \rho\left(w_{0}\right)^{-1} \rho(z)^{-1} \\
& =\sum_{z \in W} z(\mathbf{c})\left(z w_{0}\right)\left(\vec{g}_{w_{0}}\right) \chi \cdot \rho\left(z w_{0}\right)^{-1} .
\end{aligned}
$$

Suppose that the statement of the lemma is valid for all $w^{\prime}$ with $\ell\left(w^{\prime}\right)>\ell(w)$ (and for any $\vec{G} \in\left(H^{\sim}\right)^{n}$ satisfying (5.2.1)). We choose $s=s_{\alpha}, \alpha \in \Pi$, so that $\ell(w s)>\ell(w)$. Set $\vec{G}^{\prime}=\vec{G} \cdot T_{s}$. This $\vec{G}^{\prime}$ also satisfies the condition (5.2.1). Since $\vec{G}^{\prime}=\sum \vec{g}_{y} T_{y} T_{s}=\sum \vec{g}_{y}^{\prime} T_{y}$, we have $\vec{g}_{w s}^{\prime}=\vec{g}_{w}+\left(t_{\alpha}-1\right) \vec{g}_{w s}(\alpha=\alpha(s))$. By the induction hypothesis on $w s$ for $\vec{G}^{\prime}$, we see that

$$
\begin{aligned}
t_{\alpha} \vec{G} \cdot \chi= & \vec{G} \cdot T_{s} \chi=\vec{G}^{\prime} \cdot \chi \\
= & \sum_{z \in W} z(\mathbf{c})\left(z w_{0}\right)\left(\vec{g}_{w s}^{\prime}\right) \chi \cdot \rho\left(z w_{0}\right)^{-1} \\
= & \sum_{z \in W} z(\mathbf{c})\left(z w_{0}\right)\left(\vec{g}_{w}\right) \chi \cdot \rho\left(z w_{0}\right)^{-1} \\
& +\left(t_{\alpha}-1\right) \sum_{z \in W} z(\mathbf{c})\left(z w_{0}\right)\left(\vec{g}_{w s}\right) \chi \cdot \rho\left(z w_{0}\right)^{-1} .
\end{aligned}
$$

Hence we get

$$
\vec{G} \cdot \chi=\sum_{z \in W} z(\mathbf{c})\left(z w_{0}\right)\left(\vec{g}_{w}\right) \chi \cdot \rho\left(z w_{0}\right)^{-1}
$$

by the hypothesis on $w s$ for $\vec{G}$.

5.4. Let $\chi=\sum T_{w}$ be as above. Since $G \cdot \chi=\sum_{w \in W} g_{w} t_{w} \chi$ for $G=\sum_{w \in W} g_{w} T_{w} \in$ $H^{\sim}$, we see that the map $H^{\sim} \rightarrow A^{\sim}$ given by $\sum g_{w} T_{w} \rightarrow \sum g_{w} t_{w}$ gives a (left) $H^{\sim}$ homomorphism. Here the action of $H^{\sim}$ on $A^{\sim}$ is given by the formulas

$$
T_{s}(g)=t_{\alpha} s(g)+\left(\mathbf{c}_{-\alpha}-t_{\alpha}\right)(s(g)-g) \quad\left(s=s_{\alpha}, \alpha \in \Pi ; g \in A^{\sim}\right)
$$

and

$$
f(g)=f g \quad\left(f, g \in A^{\sim}\right) .
$$

Note that $J_{w}(g)=w(g)$ for $w \in W$.

5.5. Now we turn our attention to $V^{\sim}$. The $H^{\sim}$-homomorphism corresponding to the one $H^{\sim} \rightarrow A^{\sim}$ in 5.4 is the map ${ }^{0}: V^{\sim} \rightarrow A^{\sim}$ given by $F \mapsto F^{0}\left(F \in V^{\sim}\right)$ in 4.5. (Note that $V^{\sim}$ and $H^{\sim}$ are naturally isomorphic as left $H^{\sim}$-modules.) In particular, we have $\left(J_{w} F\right)^{0}=w\left(F^{0}\right)$ for $w \in W$. We regard

$$
\left(V^{\sim}\right)^{n}=\left\{\vec{F}=\left(F_{1}, \ldots, F_{n}\right) \mid F_{i} \in V^{\sim}(i=1, \ldots, n)\right\}
$$

as a left $H^{\sim}$-module (cf. 5.1). Then we can reformulate Lemma 5.4 in terms of $V^{\sim}$. 
R-Matrix Arising from Affine Hecke Algebras

Lemma 5.6 Suppose that $\vec{F}=\left(F_{1}, \ldots, F_{n}\right) \in\left(V^{\sim}\right)^{n}$ satisfies the condition

$$
J_{w} \vec{F}=\vec{F} \cdot \rho(w) \quad(w \in W) .
$$

Then we have

$$
\begin{aligned}
\vec{F}^{0} & =\left(F_{1}^{0}, \ldots, F_{n}^{0}\right) \\
& =\sum_{z \in W} z(\mathbf{c})\left(z w_{0}\right)\left(\vec{f}_{w}\right) \cdot \rho\left(z w_{0}\right)^{-1}
\end{aligned}
$$

for any $w \in W$. Here

$$
\vec{f}_{w}=\left(f_{1 w}, \ldots, f_{n w}\right) \in\left(A^{\sim}\right)^{n}(w \in W)
$$

is given by the expression

$$
F_{i}=\sum_{w \in W} f_{i w} h_{w} \in V^{\sim}(i=1, \ldots, n) .
$$

Now we are in a position to prove Theorem 4.6.

5.7. Proof of 4.6 in the case (4.2a). Let $F \in V^{\sim}$ be a solution of $\mathrm{QKZ}$ for $u \in E$;

$$
L_{\mu} F=P_{\mu}^{u} R_{\tau_{\mu}} F \quad(\forall \mu \in M)
$$

Let $\pi$ be one of the minuscule coweights of $\Delta_{*}$ as in 4.2. We set $\sigma=w_{0} w_{\pi}$ (see 4.4). We note that $\tau_{-\pi}=\sigma^{-1} \gamma$ for some $\gamma \in \Omega$ [B; Chap. 6, Sect. 2]. By 3.7 and 2.5 , we have $R_{\tau_{\pi}}=R_{\sigma}=r_{\sigma}^{-1} \cdot J_{\sigma}$. Since $J_{w}^{-1} F$ is also a solution of the above Eq. (5.7.1) for $w \in W$ (see 3.11), we have the identity (for $\mu=\pi$ ) in $A^{\sim}$,

$$
\left(\sum_{w \in W} J_{w} \mathbf{c} L_{\pi} J_{w}^{-1} F\right)^{0}=\left(\sum_{w \in W} J_{w} \mathbf{c} P_{\pi}^{u} R_{\sigma} J_{w}^{-1} F\right)^{0} .
$$

We shall calculate both sides of (5.7.2) separately. As for the left-hand side, we have

$$
\begin{aligned}
& \left(\sum_{w \in W} J_{w} \mathbf{c} L_{\pi} J_{w}^{-1} F\right)^{0}=\sum_{w \in W} J_{w} \mathbf{c} L_{\pi} J_{w}^{-1} F^{0}=\sum_{w \in W} J_{w} \mathbf{c} L_{\pi} w^{-1}\left(F^{0}\right) \\
& =\sum_{w \in W} J_{w} \mathbf{c} w^{-1}\left(L_{w(\pi)} F^{0}\right)=\sum_{w \in W} w(\mathbf{c}) L_{w(\pi)} F^{0}=W_{\pi}(t) \cdot D_{\pi} F^{0},
\end{aligned}
$$

noting that $F \rightarrow F^{0}$ is an $H^{\sim}$-homomorphism (see 5.5). Here we set $W_{\pi}(t)$ $=\sum_{w \in W} t_{w}$, the Poincaré polynomial of $W_{\pi}$ and applied the identity in [Mac2],

$$
\sum_{w \in W_{\pi}}\left(\prod_{\alpha>0,\langle\alpha, \pi\rangle=0} \mathbf{c}_{w(\alpha)}\right)=W_{\pi}(t)
$$

Next we prove that

$$
\text { The right-hand side of }(5.7 .2)=W_{\pi}(t) c_{\pi, w_{0} u} F^{0}
$$

(see 4.2 and 4.3 for the definition of $c_{\pi, w_{0} u}$ ). We may assume that there exist $\vec{F}=\left(F_{1}, \ldots, F_{n}\right) \in V^{\sim}\left(F_{1}=F\right)$ with the property

$$
J_{w} \vec{F}=\vec{F} \cdot \rho(w) \quad(\forall w \in W)
$$


for some representation $\rho: W \rightarrow G L(n, \mathbb{C})$ (see 3.11). Especially we have $J_{w} F=$ $\sum_{k} \rho_{k 1}(w) F_{k}$. Let us put $F_{k}=\sum_{w \in W} f_{k, w} h_{w}\left(f_{k, w} \in A^{\sim}\right)$. Then

$$
\begin{aligned}
& \left(\sum_{w \in W} J_{w} \mathbf{c} P_{\pi}^{u} R_{\sigma} J_{w}^{-1} F\right)^{0}=\sum_{w \in W} J_{w} \mathbf{c}\left(P_{\pi}^{u} r_{\sigma}^{-1} J_{\sigma w^{-1}} F\right)^{0} \\
& =\sum_{w \in W} J_{w} \mathbf{c} \sum_{k=1}^{n} \rho_{k 1}\left(\sigma w^{-1}\right)\left(P_{\pi}^{u} r_{\sigma}^{-1} F_{k}\right)^{0} \\
& =\sum_{w \in W} J_{w} \mathbf{c} \sum_{k=1}^{n} \rho_{k 1}\left(\sigma w^{-1}\right) \sum_{z \in \pi_{W}} q^{\langle\pi, z u\rangle} t_{z} \sum_{y \in W_{\pi}} \sigma^{-1}\left(f_{k, \sigma y z}\right) t_{y} . \\
& =\sum_{w \in W} w(\mathbf{c}) \sum_{k=1}^{n} \rho_{k 1}\left(\sigma w^{-1}\right) \sum_{z \in \pi_{W}} q^{\langle\pi, z u\rangle} t_{z} \sum_{y \in W_{\pi}}\left(w \sigma^{-1}\right)\left(f_{k, \sigma y z}\right) t_{y} .
\end{aligned}
$$

Here ${ }^{\pi} W$ denotes the set of coset representatives for $W_{\pi} \backslash W$ with minimal length. Since $\sum_{z \in \pi_{W}} q^{\langle\pi, z u\rangle} t_{z}=c_{\pi, w_{0} u}$ by 4.4 , it is sufficient to show that

$$
\sum_{w \in W} w(\mathbf{c}) \sum_{k=1}^{n} \rho_{k 1}\left(\sigma w^{-1}\right) \sum_{y \in W_{\pi}}\left(w \sigma^{-1}\right)\left(f_{k, \sigma y z}\right) t_{y}=W_{\pi}(t) \cdot F^{0}
$$

for any $z \in \in^{\pi} W$ in order to get (5.7.4).

Set $\bar{\pi}=-\sigma \pi=-w_{0} \pi$. Then $\bar{\pi}$ is also a minuscule coweight since $\left\langle\bar{\pi}, \alpha_{*}\right\rangle=$ $-\left\langle\pi, w_{0}\left(\alpha_{*}\right)\right\rangle$. Therefore we see $\sigma W_{\pi} \sigma^{-1}=W_{\bar{\pi}}$ and that $z \in \in^{\pi} W$ if and only if $\sigma z \in^{\bar{\pi}} W$. We can rewrite $(5.7 .5)$ as

$$
\sum_{w \in W} w(\mathbf{c}) \sum_{k=1}^{n} \rho_{k 1}\left(\sigma w^{-1}\right) \sum_{\bar{y} \in W_{\bar{\pi}}}\left(w \sigma^{-1}\right)\left(f_{k, \bar{y} \sigma z}\right) t_{\bar{y}}=W_{\bar{\pi}}(t) \cdot F^{0},
$$

since $t_{y}=t_{\bar{y}}$ for $\bar{y}=\sigma y \sigma^{-1} \in W_{\bar{\pi}}\left(w \in W_{\pi}\right)$. Now we note that $J_{\bar{x}} \in \sum_{\bar{y} \in W_{\bar{\pi}}} A^{\sim} \cdot T_{\bar{y}}$ for $\bar{x} \in W_{\bar{\pi}}$, which implies that

$$
\sum_{\bar{y} \in W_{\bar{\pi}}} \bar{x}\left(f_{k, \bar{y} \sigma z}\right) t_{\bar{y}}=J_{\bar{x}}\left(\sum_{\bar{y} \in W_{\bar{\pi}}} f_{k, \bar{y} \sigma z} t_{\bar{y}}\right)=\sum_{j=1}^{n} \rho_{j k}(\bar{x})\left(\sum_{\bar{y} \in W_{\bar{\pi}}} f_{j, \bar{y} \sigma z} t_{\bar{y}}\right) .
$$

Thus we have

$$
\sum_{\bar{y} \in W_{\bar{\pi}}}\left(w \sigma^{-1}\right)\left(f_{k, \bar{y} \sigma z}\right) t_{\bar{y}}=\sum_{j=1}^{n} \rho_{j k}\left(w_{\bar{\pi}}\right)\left(\sum_{\bar{y} \in W_{\bar{\pi}}}\left(w w_{0}\right)\left(f_{j, \bar{y} \sigma z}\right) t_{\bar{y}}\right)
$$

by using the expression $\sigma^{-1}=w_{0} w_{\bar{\pi}}$, where $w_{\bar{\pi}}=w_{0} w_{\pi} w_{0}$ is the longest element of $W_{\bar{\pi}}$. Substituting this in the left-hand side of (5.7.6), we finally see that (5.7.5) is equivalent to

$$
\sum_{w \in W} w(\mathbf{c}) \sum_{k=1}^{n} \rho_{j 1}\left(w_{0} w^{-1}\right) \sum_{\bar{y} \in W_{\bar{\pi}}}\left(w w_{0}\right)\left(f_{k, \bar{y} \sigma z}\right) t_{\bar{y}}=W_{\bar{\pi}}(t) \cdot F^{0},
$$

which is a consequence of Lemma 5.6. Thus we have shown that

$$
D_{\pi} F^{0}=c_{\pi, w_{0} u} F^{0}
$$


in the case (a).

5.8. Proof of 4.6 in the case (4.2b). Let $\pi$ be the short dominant coweight of $\Delta_{*}$ as in (4.2b). Since $\tau_{\pi}=s_{\alpha_{0}-n_{\alpha_{0}}} s_{\alpha_{0}}$, we have

$$
\begin{aligned}
R_{\tau_{\pi}} & =\left(r_{s_{\alpha_{0}}} \cdot R_{s_{\alpha_{0}}-n_{\alpha_{0}}} \cdot r_{s_{\alpha_{0}}}^{-1}\right) R_{s_{\alpha_{0}}} \\
& =\left(r_{s_{\alpha_{0}}} \cdot R_{-\alpha_{0}+n_{\alpha_{0}}} \cdot r_{s_{\alpha_{0}}}^{-1}\right) R_{s_{\alpha_{0}}} \\
& =R_{\alpha_{0}+n_{\alpha_{0}}} R_{\alpha_{0}}
\end{aligned}
$$

by 3.7. (Note that $H_{\alpha_{0}-n_{\alpha_{0}}}$ is the only hyperplane separating $C$ and $s_{\alpha_{0}-n_{\alpha_{0}}} C$ and that $\left(\alpha_{0}-n_{\alpha_{0}}\right)(C)<0$.) We remark that $\sigma=s_{\alpha_{0}}$ and $\bar{\pi}=\pi$ in this case if we set $\sigma=w_{0} w_{\pi}$ and $\bar{\pi}=-w_{0}(\pi)$ as in 5.7. (This comes from the fact $w_{0}=-1$.)

Let $F \in V^{\sim}$ be the solution of $\mathrm{QKZ}$ for $u \in E$,

$$
L_{\mu} F=P_{\mu}^{u} R_{\tau_{\mu}} F \quad(\forall \mu \in M) .
$$

As in the proof of the case (a) in 5.7, we have the identity (for $\mu=\pi$ ) in $A^{\sim}$,

$$
\left(\sum_{w \in W} J_{w} \mathbf{c c}_{\alpha_{0}+n_{\alpha_{0}}}\left(L_{\pi}-1\right) J_{w}^{-1} F\right)^{0}=\left(\sum_{w \in W} J_{w} \mathbf{c c}_{\alpha_{0}+n_{\alpha_{0}}}\left(P_{\pi}^{u} R_{\sigma}-1\right) J_{w}^{-1} F\right)^{0}
$$

Exactly as in 5.7, we can see that the left-hand side of (5.8.2) is equal to $W_{\pi}(t) \cdot D_{\pi} F^{0}$ (see (4.2b) for the definition of $D_{\pi}$ in this case).

Now let us calculate the right-hand side of (5.8.2). We assume as in 5.7 that the condition $J_{w} \vec{F}=\vec{F} \cdot \rho(w)(\forall w \in W)$ holds for $\vec{F}=\left(F_{1}, \ldots, F_{n}\right)$ with $F_{1}=F$. By 3.5, we have

$$
\begin{aligned}
& \mathbf{c}_{\alpha_{0}+n_{\alpha_{0}}} R_{\alpha_{0}+n_{\alpha_{0}}} h_{y} \\
& \quad=h_{y}+q^{\langle\pi, y u\rangle}\left(\mathbf{c}_{\alpha_{0}+n_{\alpha_{0}}}-t_{\alpha_{0}}\right) h_{\sigma y} \quad \text { if } y^{-1} \alpha_{0}>0 \\
& =t_{\alpha_{0}} h_{y}+q^{\langle\pi, y u\rangle}\left(\mathbf{c}_{\alpha_{0}+n_{\alpha_{0}}}-1\right) h_{\sigma y} \quad \text { if } y^{-1} \alpha_{0}<0 .
\end{aligned}
$$

Since

$$
R_{\sigma} J_{w}^{-1} F=r_{\sigma}^{-1} J_{\sigma w^{-1}} F=\sum_{w \in W} \sum_{k=1}^{n} \rho_{k 1}\left(\sigma w^{-1}\right) \sigma^{-1}\left(f_{k, \sigma w}\right) h_{w}
$$

we have

$$
\begin{aligned}
\mathbf{c}_{\alpha_{0}+n_{\alpha_{0}}} & P_{\pi}^{u} R_{\tau_{\mu}} J_{w}^{-1} F \\
= & \mathbf{c}_{\alpha_{0}+n \alpha_{0}} P_{\pi}^{u} R_{\alpha_{0}+n_{\alpha_{0}}} R_{\sigma} J_{w}^{-1} F \\
= & \sum_{k=1}^{n} \rho_{k 1}\left(\sigma w^{-1}\right) \sum_{y \in W, y^{-1}\left(\alpha_{0}\right)>0}\left[\sigma^{-1}\left(f_{k, \sigma y}\right)\left\{q^{\langle\pi, y u\rangle} h_{y}+\left(\mathbf{c}_{\alpha_{0}+n_{\alpha_{0}}}-t_{\alpha_{0}}\right) h_{\sigma y}\right\}\right. \\
& \left.+\sigma^{-1}\left(f_{k, y}\right)\left\{q^{-\langle\pi, y u\rangle} t_{\alpha_{0}} h_{\sigma y}+\left(\mathbf{c}_{\alpha_{0}+n \alpha_{0}}-1\right) h_{y}\right\}\right] .
\end{aligned}
$$

Hence, if we put

$$
G_{w}=\sum_{k=1}^{n} \rho_{k 1}\left(\sigma w^{-1}\right) \sum_{w \in W} \sigma^{-1}\left(f_{k, y}\right) h_{y}
$$


for $w \in W$, we have

$$
\begin{aligned}
& \mathbf{c}_{\alpha_{0}+n_{\alpha_{0}}}\left(P_{\pi}^{u} R_{\tau_{\mu}} J_{w}^{-1} F-G_{w}\right) \\
& =\sum_{k=1}^{n} \rho_{k 1}\left(\sigma w^{-1}\right) \sum_{y \in W, y^{-1}\left(\alpha_{0}\right)>0}\left[\sigma^{-1}\left(f_{k, \sigma y}\right)\left\{q^{\langle\pi, y u\rangle} h_{y}-t_{\alpha_{0}} h_{\sigma y}\right\}\right. \\
& \left.\quad+\sigma^{-1}\left(f_{k, y}\right)\left\{q^{-\langle\pi, y u\rangle} t_{\alpha_{0}} h_{\sigma y}-h_{y}\right\}\right] .
\end{aligned}
$$

Note that

$$
\left\{y \in W \mid y^{-1}\left(\alpha_{0}\right)>0\right\}=\left\{x z \in W \mid x \in W_{\pi}, z \in \in^{\pi} W, z^{-1}\left(\alpha_{0}\right)>0\right\},
$$

and that $\sigma z \in \in^{\pi} W$ if and only if $z \in{ }^{\pi} W$. Therefore the same argument as in 5.7 using Lemma 5.6 shows that

$$
\begin{aligned}
& \left(\sum_{w \in W} J_{w} \mathbf{c c}_{\alpha_{0}+n_{\alpha_{0}}}\left(P_{\pi}^{u} R_{\tau_{\mu}} J_{w}^{-1} F-G_{w}\right)\right)^{0} \\
& \quad=\sum_{y \in W, y^{-1} \alpha_{0}>0}\left\{q^{\langle\pi, y u\rangle} t_{y}-t_{\alpha_{0}} t_{\sigma y}+q^{-\langle\pi, y u\rangle} t_{\alpha_{0}} t_{\sigma y}-t_{y}\right\} F^{0} \\
& \quad=W_{\pi}(t) c_{\pi, w_{0} u} F^{0}
\end{aligned}
$$

(see 4.4 for the expression of $c_{\pi, w_{0} u}$ ). But

$$
G_{w}^{0}=\sigma^{-1}\left(J_{\sigma^{-1}} F\right)^{0}=\left(J_{w} F\right)^{0}
$$

by 5.5 . Thus we finally have

$$
\left(\sum_{w \in W} J_{w} \mathbf{c c}_{\alpha_{0}+n_{\alpha_{0}}}\left(P_{\pi}^{u} R_{\tau_{\mu}}-1\right) J_{w}^{-1} F\right)^{0}=W_{\pi}(t) c_{\pi, w_{0} u} F^{0},
$$

and complete the proof of Theorem 4.6.

Acknowledgement. We are grateful to I. Cherednik and A. Matsuo for raising our interest in this subject. Moreover Cherednik made useful comments on the first version of this paper, which we greatly acknowledge. We also would like to thank E. Date and M. Jimbo for their helpful comments on the R-matrix and QKZ.

\section{References}

[B] Bourbaki, N.: Groupes et algèbres de Lie, Chap. IV, V, VI. Paris: Hermann, 1968

[C1] Cherednik, I.: Factorized particles on the half-line and root systems. Theor. Math. Phys. 61, 35-43 (1984)

[C2] Cherednik, I.: A unification of Knizhnik-Zamolodchikov and Dunkle operators via affine Hecke algebras. Invent. Math. 106, 411-432 (1991)

[C3] Cherednik, I.: Integration of quantum many body problems by affine KnizhnikZamolodchikov equations. Preprint, RIMS.

[C4] Cherednik, I.: Quantum Knizhnik-Zamolodchikov equations and affine root systems. Commun. Math. Phys. 150, 109-136 (1992)

[C5] Cherednik, I.: Double affine Hecke algebras and Macdonald's operators. Internat. Math. Res. Notices No. 9, 171-180 (1992) 
[FR] Frenkel, I.B., Reshetikhin, N.Yu.: Quantum affine algebras and holonomic difference equations. Commun. Math. Phys. 146, 1-60 (1992)

[J] Jimbo, M.: A $q$-analogue of $U(\mathfrak{g l}(\mathrm{N}+1))$, Hecke algebra, and the Yang-Baxter equation. Lett. Math. Phys. 11, 247-252 (1986)

[K] Kato, S.: On the Kazhdan-Lusztig polynomials for affine Weyl groups. Adv. in Math. 55, $103-130$ (1985)

[K2] Kato, S.: On eigenspaces of the Hecke algebra with respect to a good maximal compact subgroup of a $p$-adic reductive group. Math. Ann. 257, 1-7 (1981)

[K3] Kato, S.: Irreducibility of principal series representations for Hecke algebras of affine type. J. Fac. Sci. Univ. Tokyo Sec. IA 28, 929-943 (1982)

[L] Lusztig, G.: Affine Hecke algebras and their graded version. J. Am. Math. Soc. 2, 599-635 (1989)

[Mac1] Macdonald, I.G.: Affine root systems and Dedekind's $\eta$-function. Invent. Math. 15, 91-143 (1972)

[Mac2] Macdonald, I.G.: Poincaré series for Coxeter groups. Math. Ann. 119, 161-174 (1972)

[Mac3] Macdonald, I.G.: Orthogonal polynomials associated with root systems. Preprint.

[Matm] H. Matsumoto: Analyse harmonique dans les systèmes de Tits bornologiques de type affine. Lect. Notes in Math. 590, Berlin Heidelberg New York: Springer, 1977

[Mato] Matsuo, A.: Integrable connections related to zonal spherical functions. Invent. Math. 110, 95-121 (1992)

Communicated by N. Yu. Reshetikhin 
